\title{
Educação e envelhecimento: contribuições da perspectiva Life-Span
}

\author{
Education and aging: Life-Span \\ perspective contributions
}

\author{
Natália Nunes SCORALICK-LEMPKE' \\ Altemir José Gonçalves BARBOSA'
}

\section{Resumo}

A perspectiva Life-Span é um importante marco teórico no estudo do envelhecimento, uma vez que colaborou para mudar a concepção de que o idoso é um ser passivo e doente, ressaltando a possibilidade de desenvolvimento durante todo o curso da vida. Também destacou a heterogeneidade na velhice, enfatizando a importância de atividades para a manutenção do envelhecimento saudável. A aquisição de novas aprendizagens tem sido considerada uma tarefa importante nesse sentido, uma vez que pode otimizar as capacidades cognitivas e favorecer a rede de suporte social do idoso. Assim, este trabalho, a partir da apresentação dos pressupostos que conferiram extrema relevância à Life-Span no âmbito da Psicologia do Desenvolvimento, discute a importância da educação para a velhice saudável, a oferta de atividades educacionais para idosos no Brasil e elucida a amplitude de termos encontrados na literatura para designar o processo de aprendizagem ao longo do curso da vida.

Unitermos: Desenvolvimento humano. Educação. Envelhecimento.

\begin{abstract}
Life-Span perspective is an important theoretical milestone in the study of aging, since it helped to change the concept that the elderly person is a passive and sick being, by highlighting the possibility of development throughout the course of life. It has also stressed the heterogeneity of old age, emphasizing the importance of activities in order to maintain healthy aging. The acquisition of new learning has been considered an important task in this sense, since it may enhance the cognitive skills and favor the social support network for the elderly. Thus, by the presentation of assumptions that give the utmost importance to Life-Span in the field of Developmental Psychology, the present study discusses the importance of education for a healthy old age, the offer of educational activities for the elderly in Brazil and elucidates the range of terms found in the literature to describe the learning process throughout the course of life.
\end{abstract}

Uniterms: Human development. Education. Aging.

$\boldsymbol{\nabla \nabla v \boldsymbol { v }}$

1 Universidade Federal de Juiz de Fora, Instituto de Ciências Humanas, Departamento de Psicologia. R. José Lourenço Kelmer, s/n., Campus Universitário São Pedro, 36036-330, Juiz de Fora, MG, Brasil. Correspondência para/Correspondence to: N.N. SCORALICK-LEMPKE. E-mail: <nataliascoralick@yahoo.com.br>.

Artigo elaborado a partir da dissertação de N.N. SCORALICK-LEMPKE, intitulada "Alfabetização digital: aprendizagem e utilização do computador na velhice". Universidade Federal de Juiz de Fora, 2009. Apoio: Fundação de Apoio à Pesquisa do Estado de Minas Gerais (Processo no SHA APQ 5892-5.0607) e Coordenação de Aperfeiçoamento de Pessoal de Nível Superior. 
O aumento populacional de indivíduos com idade igual ou superior a 60 anos é um fenômeno mundial e tem ocorrido de forma acelerada, principalmente nos países em desenvolvimento. De acordo com o Instituto Brasileiro de Geografia e Estatística - IBGE (2008), a população brasileira apresentou um crescimento de 10,5\% no período de 1997 a 2007, ao passo que, no contingente populacional de idosos, esse aumento foi de $47,8 \%$. Essa constatação evidencia que o envelhecimento populacional torna-se cada vez mais relevante, superando bastante o aumento da população total.

O crescimento da população de idosos despertou a atenção de pesquisadores de diversas áreas, de forma que as últimas décadas do século XX, especificamente, foram marcadas por uma progressiva produção sobre o tema (Papaléo Netto, 2002). Os estudos bibliométricos de Minayo, Souza e Paula (2010) e de Ravelli et al. (2009) são exemplos de pesquisas que, contemplando diferentes temas relacionados ao envelhecimento, destacam a ampliação da produção científica sobre a velhice. Esse fato, associado a mudanças políticas, sociais e culturais, contribuíram para alterar a concepção de que o envelhecimento estaria associado somente ao declínio, à doença e à incapacidade. Nesse contexto, destaca-se a perspectiva Life-Span.

A perspectiva Life-Span é uma abordagem de orientação dialética que tem colaborado expressivamente para as mudanças de paradigma acerca da velhice (Baltes, 1987; P.B. Baltes \& M.M. Baltes, 1990). O empenho de Paul P. Baltes e, consequentemente, sua extensa obra sobre o tema tornaram-no um dos autores mais expressivos dessa proposta no âmbito da Psicologia do Desenvolvimento, principalmente no que se refere ao desempenho intelectual dos idosos (Neri, 2001; 2006). Transcorridas mais de duas décadas das proposições iniciais de Baltes, é possível afirmar, ainda, que, no Brasil, elas podem ser consideradas como uma concepção emergente.

A partir de uma gama de pressupostos sobre o desenvolvimento humano, a perspectiva Life-Span concebe o envelhecimento como um processo multideterminado e heterogêneo, que pode ser categorizado em três tipos: normal, referindo-se às alterações típicas e inevitáveis ao envelhecimento; patológico, em que se 648 encontram os casos de doenças, disfuncionalidade e descontinuidade do desenvolvimento; e ótimo ou saudável, caracterizado por um ideal sociocultural de excelente qualidade de vida, funcionalidade física e mental, baixo risco de doenças e incapacidade, bem como engajamento ativo com a vida (Baltes, 1987). Apesar da proposição de três tipos distintos, é importante destacar a necessidade de não se interpretar a tipologia de forma rígida, pois esses estados podem-se sobrepor em certos momentos do curso de vida. Entretanto, é preciso reconhecê-los como indicadores da condição geral de saúde. Caso contrário, ter-se-ia um estado mítico de'nirvana', com ausência total de sofrimento e de problemas de saúde.

O envelhecimento saudável está associado à ideia de que o indivíduo preserva seu potencial de desenvolvimento durante todo o curso da vida (P.B. Baltes \& M.M. Baltes, 1990), havendo um equilíbrio entre suas limitações e potencialidades, as quais podem ser otimizadas por meio de intervenções. Uma delas se refere à aquisição de novas aprendizagens, o que tem sido destacada por diversos estudos como uma atividade que auxilia no bom funcionamento físico, psicológico e social na velhice (P.B. Baltes \& M.M. Baltes, 1990; Duay \& Bryan, 2006; Webber \& Celich, 2007).

Tendo em vista essa breve apresentação da perspectiva, o presente trabalho tem como objetivo elucidar os pressupostos básicos da Life-Span. Ainda que de forma inicial, analisa a importância da educação para a manutenção do envelhecimento saudável, discute a amplitude de termos encontrados na literatura para designar o processo de aprendizagem ao longo do curso da vida e propõe uma reflexão sobre a oferta de atividades educacionais para idosos no Brasil.

\section{A perspectiva Life-Span e a educação na velhice}

Elaborada dentro de um campo interdisciplinar de estudos, um dos pressupostos básicos da perspectiva Life-Spané que o desenvolvimento psicológico também necessita ser estudado em um contexto que englobe múltiplas disciplinas, como a Antropologia, a Biologia e a Sociologia (Baltes, Resse \& Lipsitt, 1980). Baltes (1987) afirma que a multiplicidade de influências sobre as origens e direções do desenvolvimento ao longo do curso da vida está intrinsecamente relacionada ao fato de que qualquer disciplina isolada sobre ele é incom- 
pleta. Dessa forma, estudá-lo dentro de um único campo do conhecimento humano ofereceria uma compreensão parcial do curso vital.

Segundo Baltes et al. (1980) e Baltes (1987), o desenvolvimento psicológico é um processo que se estende por toda a vida. Nenhuma condição especial de maturidade é assumida como princípio geral, assim como nenhum período no curso da vida detém supremacia na regulação da natureza do desenvolvimento. Portanto, em todas as fases, o ser humano pode desenvolver habilidades que o auxiliem em sua capacidade adaptativa. Segundo Neri (1995), a aquisição, a manutenção, o aperfeiçoamento e a extinção dos comportamentos sociais e cognitivos são processos que podem originar-se em qualquer etapa, uma vez que nem todas as mudanças do desenvolvimento estão ligadas à idade.

De acordo com a perspectiva Life-Span, o desenvolvimento e a manutenção de padrões efetivos de envelhecimento não somente dependem de determinantes de natureza genético-biológica, mas também são influenciados por fatores socioculturais (Baltes et al., 1980; Baltes, 1987). Assim, para que o desenvolvimento se estenda até idades mais avançadas, é necessária a disponibilidade de recursos culturais. A oferta de programas que forneçam atividades e estímulos para os idosos parece ser, portanto, importante nessa fase da vida, a fim de que eles possam desfrutar de um envelhecimento equilibrado entre os declínios, decorrentes do próprio processo, e os benefícios, que podem ser proporcionados através dessas estratégias.

Baltes et al. (1980) e Baltes (1987) afirmam que o desenvolvimento individual pode ser compreendido como o resultado da interação entre três tipos de influências: graduadas pela idade; graduadas pela história; e não normativas. A primeira se refere aos determinantes genético-biológicos e ambientais, que têm uma forte relação com a idade cronológica e tendem a ocorrer de modo semelhante para todos os indivíduos de uma mesma cultura. A maturação biológica e os eventos sociais determinados pela idade fazem parte desse grupo. As influências normativas graduadas pela história, por sua vez, consistem em fatores psicossociais, associados ao tempo e ao contexto histórico de uma determinada coorte, os quais geralmente acontecem de forma similar para a maioria dos membros. Para elucidar esse tipo de influência, Baltes (1987) cita a vivência de uma guerra por um grupo de pessoas. O último tipo de influência, isto é, as não normativas, diz respeito aos determinantes biológicos e ambientais que não têm caráter universal, sendo que sua ocorrência não é previsível em matéria de época ou sequência para indivíduos ou para grupos. Como exemplo, menciona-se o acometimento de determinada doença ou a perda de um dos genitores na infância. Ademais, cabe destacar que, em cada tipo de influência, há uma interação entre fatores biológicos e ambientais, que resultam nas diferenças individuais.

Outro pressuposto fundamental da perspectiva Life-Span diz respeito à multidirecionalidade do desenvolvimento (Baltes et al., 1980; Baltes, 1987). De acordo com os autores, mudanças podem assumir diferentes direções num mesmo período do desenvolvimento, incluindo crescimento em determinados domínios e declínio em outros.

Relacionado à concepção de multidirecionalidade, encontra-se o pressuposto de que o desenvolvimento se constitui a partir da dinâmica entre crescimento (ganhos) e declínio (perdas) (Baltes, 1987). Assim como em outras fases da existência, na velhice há um equilíbrio entre ganhos e perdas, influenciado pelo modo como cada pessoa organiza seu curso de vida, pelos fatores socioculturais e pela incidência de diferentes patologias, caracterizando a heterogeneidade dessa fase. Os ganhos e perdas nas diversas etapas da vida seguem uma proporção diferente, predominando-se os declínios na velhice.

No que diz respeito ao desenvolvimento intelectual, Baltes (1987) afirma que, no envelhecimento, há uma perda nas capacidades cognitivas decorrentes do baixo funcionamento neurológico, sensorial e psicomotor, o que reflete na capacidade de adaptação do indivíduo. Ressalta, no entanto, que pode haver mudanças qualitativas na vida adulta e na velhice que irão depender muito mais das oportunidades oferecidas pela cultura do que dos mecanismos genético-biológicos.

Outro conceito central da perspectiva Life-Span é o de plasticidade, que se refere ao potencial de mudança de um indivíduo e à sua flexibilidade para lidar com novas situações (Baltes, 1987). O grau de plasticidade é consoante à capacidade de reserva da pessoa, constituída por recursos internos e externos que mudam 
de acordo com o tempo e com a situação (Staudinger, Marsiske \& Baltes, 1993). Assim, as limitações decorrentes do envelhecimento podem ser minimizadas pela ativação dessas capacidades, dependendo do grau de plasticidade individual (Baltes, 1987).

Os pressupostos anteriormente descritos contribuíram para que a perspectiva Life-Span se tornasse a corrente dominante na psicologia do envelhecimento (Neri, 2006). Um dos pontos principais dessa perspectiva é a concepção de que o desenvolvimento das capacidades cognitivas ocorre durante toda a vida, inclusive na velhice, contrapondo-se à tradicional ideia de que somente crianças e adolescentes poderiam se desenvolver cognitivamente.

Em consonância, o pressuposto de que, em todo o desenvolvimento, existe a possibilidade de crescimento e de declínio das capacidades adaptativas contribuiu para reverter a concepção de que a velhice estaria associada somente a perdas. Estudos sobre a inteligência na idade adulta vêm confirmando esses achados ao demonstrar que, a partir de treino cognitivo, indivíduos idosos podem melhorar seu desempenho (Aramaki \& Yassuda, 2011; Brum, Forlenza \& Yassuda, 2009; Fillit et al., 2002; Lima-Silva et al., 2010; Schaie, 1979). A participação em ambientes estimulantes e a presença de oportunidades de desenvolvimento têm-se demonstrado fundamentais para um melhor desempenho intelectual ao longo da vida (Schaie, 1979; Yassuda \& Silva, 2010).

Destaca-se, ainda, a importância atribuída pela perspectiva Life-Span à influência dos fatores socioculturais no processo de desenvolvimento. A criação de oportunidades de lazer, de socialização e, principalmente, de educação mostra-se fundamental nessa fase da vida, permitindo equilibrar os declínios inerentes ao envelhecimento e os benefícios proporcionados por essas atividades.

\section{A importância da educação na velhice}

Palma e Cachioni (2002) ressaltam que é extremamente significativa a procura, por parte dos idosos, por atividades educacionais em programas oferecidos em universidades, associações e sindicatos, em cursos de línguas, de formação profissional e de reciclagem, 650 em sistemas de aprendizagem aberta e de formação à distância. De acordo com as autoras, em países como a Suécia e o Japão, as taxas de participação da população de adultos e de idosos nessas atividades situam-se em torno de 50\%, evidenciando, portanto, o interesse e a motivação desse segmento da população em adquirir novos conhecimentos, aumentar sua rede de suporte social e/ou construir suas próprias trajetórias.

Diversos estudos sobre a educação têm demonstrado a importância de atividades desse tipo para o desenvolvimento de habilidades na velhice e para a manutenção do envelhecimento saudável. Ordonez, Yassuda e Cachioni (2011) verificaram que um grupo de 22 idosos, participantes de um programa de inclusão digital constituído por 15 encontros de 120 minutos cada, apresentou um aumento no desempenho cognitivo, especialmente na linguagem e memória, quando comparados a um grupo controle.

Em um estudo transversal com 184 idosos participantes da Universidade Aberta à Terceira Idade da Escola de Artes, Ciências e Humanidades da Universidade de São Paulo, Batistoni et al. (2011) verificaram uma associação entre o tempo de participação nas atividades e o menor índice de sintomas depressivos. Assim, a participação em atividades educacionais, sociais e físicas serviu como um possível fator protetor para a depressão entre os idosos que estiveram incluídos no programa por mais de um semestre (Batistoni et al., 2011). Portero e Oliva (2007), em estudo longitudinal com 147 idosos participantes da Universidade da Terceira Idade de Sevilha - Espanha, também observaram que as atividades contribuíram para um aumento do suporte social, gerando, por conseguinte, aumento no senso de bem-estar e melhoria na saúde geral dos participantes.

Duay e Bryan (2006) afirmam que, além de ser uma forma de exercitar a mente, a aquisição de aprendizagens na velhice permite novas experiências sociais, funcionando como uma estratégia de enfrentamento frente às perdas que ocorrem nessa fase da vida e como uma forma de lazer e obtenção de prazer. Segundo Webber e Celich (2007), a educação de idosos permite uma ressignificação das experiências anteriores à velhice e, principalmente, das vivências experimentadas durante o curso de vida. Assim, o envelhecimento assume significados diferentes, permitindo que o idoso reveja seu projeto de vida, seus ideais e expectativas, 
fazendo com que experimente maior liberdade, expresse-se de forma autônoma e exerça sua cidadania.

Swindell eThompson (1995) elucida cinco razões que justificam a importância da educação para essa coorte etária: 1) pode ajudar os idosos a terem mais autoconfiança e independência, reduzindo as possibilidades de dependência de recursos públicos e privados; 2) é fundamental na capacitação dos idosos para lidarem com os inumeráveis problemas práticos e psicológicos em um mundo complexo, fragmentado e em mudanças; 3 ) intensifica a atuação e a contribuição para a sociedade; 4) é possível aumentar o autoconhecimento, compreender-se melhor e comunicar as próprias experiências às outras gerações, o que favorece o equilíbrio, as perspectivas pessoais e de mundo, qualidades valiosas em uma sociedade em mudança; 5) é crucial para muitos idosos motivados para a aprendizagem e para a comunicação.

Os programas educacionais para idosos também funcionam como instrumento para prolongar o processo de socialização que se inicia na infância e vai até a velhice. De acordo com Cachioni (1998), se na infância e adolescência a atualização dos valores e normas ocorre especialmente na escola, na velhice a educação é concebida como oportunidade de atualização, aquisição de conhecimentos e participação em atividades culturais, sociais, políticas e de lazer. Além de desenvolver e manter as habilidades cognitivas, a aprendizagem nessa fase da vida pode ser um grande facilitador da socialização (Duay \& Bryan, 2008).

Posada (2004) afirma que a educação pode ser uma estratégia para potencializar e, também, para compensar os possíveis prejuízos nas habilidades cognitivas dos idosos. Assim, a educação pode estimular o crescimento e a aquisição de novas competências, fomentando possíveis ganhos durante toda a vida. Além disso, ela representa uma via de remediação e retomada dos níveis de funcionamento anteriores, tendo três diferentes funções: 1) oferecer recursos àqueles que, por circunstâncias pessoais, não tiveram oportunidades educativas em etapas anteriores; 2) auxiliar os idosos quanto à prevenção e à solução de déficits associados a essa fase, na medida em que novos aprendizados propiciam significativos benefícios sobre o funcionamento cognitivo; 3) facilitar a retomada de papéis sociais, bem como potencializar as atividades e os relacionamentos interpessoais, evitando as perdas dos vínculos que parte da população idosa experimenta.

Além disso, a educação pode reduzir a dependência dos idosos em relação aos programas sociais do estado e aumentar sua qualidade de vida (Duay \& Bryan, 2008). Para Veras (2009), em menos de 40 anos, o Brasil passou de um cenário de mortalidade, próprio de um País jovem, para um quadro de enfermidades complexas e onerosas, típicas dos países longevos. Segundo o autor, as alterações biológicas e cognitivas características do envelhecimento patológico poderiam ser amenizadas por meio de políticas públicas de promoção e prevenção de saúde, dentre as quais se incluem as atividades educacionais, gerando menores custos com hospitalização e serviços de reabilitação. Reitera-se, ainda, que a educação traz benefícios diretos aos idosos, auxiliando na manutenção e prevenção da saúde, o que contribui para o envelhecimento saudável.

Os benefícios sociais, cognitivos e afetivos da educação para idosos apresentados anteriormente são científica, social e moralmente incontestáveis. Não há uma garantia, contudo, de que eles ocorrerão em todas as situações de aprendizagem e que atingirão todos os envolvidos. Do mesmo modo que na educação de outras coortes etárias, alcançar o objetivo educacional depende em grande medida da qualidade do ato de ensino, que, para ser elevada, exige, dentre outros aspectos, uma concepção sólida de desenvolvimento na velhice como a proposta pela perspectiva Life-Span.

\section{Educação na velhice: um campo em construção}

No final da década de 1990, Cachioni (1998) destacava que, apesar de existirem iniciativas educacionais para pessoas com mais de 60 anos e estudos comprovando seus benefícios, havia também uma variedade de termos para designar o processo de aprendizagem ao longo do curso da vida, o que evidenciava a prematuridade dos estudos da área. Mais de uma década depois, é possível afirmar que, especialmente no Brasil, a pluralidade terminológica persiste.

Uma das expressões frequentemente usadas nesse contexto é educação continuada. De acordo com Todaro (2005), a expressão faz referência às atividades de ensino que ocorrem após o término do processo formal de educação e de formação profissional, que 
tem por finalidade a atualização e a aquisição de informações de forma sistemática. Elas permitem que o trabalhador mantenha ou melhore suas habilidades e, consequentemente, continue inserido no mercado de trabalho.

Outra expressão bastante encontrada na literatura é educação permanente. Decorrente da necessidade de acompanhar as rápidas transformações ocorridas na atualidade, trata-se de uma educação diferente da formal, já que engloba aspectos econômicos, políticos e culturais, atingindo diversos setores da convivência humana (Todaro, 2005). A partir desse conceito, a educação é vista como algo que se prolonga durante toda a existência, não havendo distinção entre o tempo escolar, a preparação para a vida e o tempo para o exercício profissional:"a educação deixa de ser mera aquisição de conhecimentos para transformar-se em processo de desenvolvimento, por meio das experiências vividas ao longo da existência" (Todaro, 2005, p.64).

Cachioni (1998) destaca que o desenvolvimento pessoal é um dos objetivos da educação permanente. Ela abre novos horizontes, oferta condições para que se experimente maior liberdade, permite que as pessoas sintam e expressem sua personalidade de forma mais autônoma e mais autêntica e, ainda, possibilita que se relacionem melhor com os outros e com o mundo. Assim, a educação permanente é uma questão vital para a continuidade do processo de desenvolvimento por permitir que o indivíduo continue ativo e participante em sua comunidade.

No caso específico da população com mais de 60 anos, a expressão gerontologia educacional também vem sendo muito empregada (Cachioni, 1998). Utilizada pela primeira vez por David A. Peterson, foi definida como "um campo de estudo e prática de métodos e técnicas de ensino, numa tentativa de integrar as instituições e processos de educação com o conhecimento do envelhecer e as necessidades do idoso" (Cachioni, 1998, p.20). De acordo com Lima (2000), trata-se de uma pedagogia específica para essa população, que contempla interesses, motivações e características próprias de ensino. Pressupõe que os idosos sejam capazes não só de aprender e administrar sua vida competentemente, mas também de continuar a trazer contribuições significativas e produtivas a sua comunidade (Cachioni,

652 2007).Também objetiva promover melhor qualidade de vida aos idosos, na medida em que explora suas capacidades e previne o declínio físico, psicológico e social (Cachioni, 1998).

Conforme citado por Cachioni (2005), a gerontologia educacional é um campo interdisciplinar que compreende três áreas: 1) educação para os idosos, com programas educacionais voltados a atender às necessidades dessa população, considerando as características dessa coorte etária; 2) educação para a população em geral sobre a velhice e os idosos, com programas educacionais, como um espaço intergeracional, que possibilita à população mais jovem rever seus conceitos sobre a velhice e o seu próprio processo de envelhecimento; 3) formação de recursos humanos, por meio da capacitação técnica de profissionais para a prestação de serviços direcionados à pessoa idosa e à formação de pesquisadores.

Posteriormente e a partir dessa divisão, Glendenning (1989) propôs as seguintes categorias com focos distintos para definir os processos de ensino associados ao envelhecimento: gerontologia educacional, que objetiva os processos de aprendizagem dos adultos maduros e idosos; e educação gerontológica, cuja finalidade é preparar e atualizar profissionais em gerontologia, cuidadores informais, além de oferecer à sociedade informações sobre velhice, processo de envelhecimento e necessidades dos idosos.

Recentemente, Limieux e Martinez (2000) propuseram que o termo gerontagogia é o mais adequado para se referir ao processo educacional na velhice. Ele pode ser definido como uma ciência educacional interdisciplinar que visa ao estudo do idoso em situação pedagógica, tratando-se, portanto, de uma ciência aplicada. Segundo os autores, o termo é mais pertinente para designar a educação voltada para o idoso, uma vez que enfatiza o processo de ensino-aprendizagem. Para eles, assim como a pedagogia tem como base teórica a psicologia educacional, a gerontagogia tem a gerontologia educacional como fundamento.

Verifica-se, consequentemente, que, apesar de não haver um consenso sobre o tema, a variedade de termos encontrados nas publicações científicas evidencia a prematuridade dos estudos, bem como o crescente interesse dos pesquisadores por esse campo de pesquisa. Independentemente da nomenclatura utilizada, ademais, deve-se considerar que o processo 
educacional na velhice adquire importância crescente na atualidade, dados os benefícios que podem advir dessas atividades, demandando, portanto, esforços para que, cada vez mais, possam ser criadas oportunidades educativas que sejam adequadas às limitações e aos interesses dos idosos. Isso implica, entre outros aspectos, uma formação adequada dos profissionais que irão ensinar essa população, materiais e métodos de ensino adaptados e um maior envolvimento dos idosos no processo de ensino-aprendizagem.

\section{Iniciativas educacionais para idosos no Brasil}

Como discutido anteriormente, a aquisição de novas aprendizagens tem sido destacada como uma atividade responsável pelo envelhecimento saudável (P.B. Baltes \& M.M. Baltes, 1990; Boulton-Lewis, Buys, Lovie-Kitchin, Barnett \& David, 2007; Russel, 2008; Webber \& Celich, 2007). Apesar disso, observa-se que ainda é pequeno o número de iniciativas educacionais para essa população, principalmente no Brasil, onde elas se restringem a praticamente duas possibilidades: educação de jovens e adultos e universidades abertas para a terceira idade.

A Lei de Diretrizes e Bases da Educação Nacional (LDB) assegura a educação aos jovens e adultos que não tiveram oportunidade de finalizar seus estudos na idade apropriada (Lei no 9.394, Brasil, 1996). A retomada dos estudos é realizada por meio do programa Educação de Jovens e Adultos (EJA), o qual busca promover a educação das pessoas que, por circunstâncias pessoais, não puderam terminar seus estudos no período desejado (Brasil, 2000). Trata-se, portanto, de um programa compensatório que permite aos estudantes, incluindo evidentemente os idosos, concluir o ensino fundamental e/ou o ensino médio em idades 'incomuns'.

Os resultados dessa política pública de educação não têm sido, no entanto, satisfatórios. Dentre outros problemas, como, por exemplo, a formação docente (Marques \& Pachane, 2010), Di Pierro (2010) alerta para o fato de que uma pequena parcela dos jovens, adultos e idosos tem efetivamente procurado os cursos disponíveis. No caso específico dos idosos, as atividades da EJA nem sempre correspondem ao que eles almejam. Não raro, os idosos descartam a necessidade de se alfabetizar ou terminar os estudos, em favor de atividades que preencham o tempo livre, propiciem novas amiza- des e permitam atualizar e/ou adquirir novas aprendizagens sem necessariamente obter um diploma (Cachioni, 1998).

Uma alternativa para idosos que não pretendem colar grau são as universidades abertas para a terceira idade. Como o próprio nome sugere, elas são vinculadas à educação superior sem, contudo, ofertar cursos de graduação ou pós-graduação. No contexto brasileiro, geralmente são fornecidos programas de educação permanente ou de educação continuada (Neri \& Cachioni, 1999).

O número dessas instituições tem aumentado desde a década de 1990 (Cachioni, 1998; 2008), provavelmente, em decorrência do crescimento do interesse dos idosos por atividades educacionais informais, como cursos de informática, artesanato e prevenção à saúde, por exemplo. As universidades para a terceira idade têm contribuído positivamente para o bem-estar de idosos e predizem, em potencial, uma velhice bem-sucedida (Irigaray \& Schneider, 2008). Todavia, tendem a atender uma pequena parcela da população, pois, em sua maioria, têm pré-requisitos formais (níveis de escolarização, mensalidades etc.) ou informais (padrões de comportamento etc.). Acabam por serem, desse modo, elitistas.

A escassez de atividades educacionais para idosos contrasta com o que é previsto no Estatuto do Idoso (Lei n 10.741, Brasil, 2003). Ele dispõe três artigos específicos sobre a educação: o Artigo 20 elucida que o idoso tem direito à educação, respeitando sua peculiar condição de saúde; o Artigo 21 estabelece que "o poder público criará oportunidades de acesso do idoso à educação, adequando currículos, metodologias e material didático aos programas educacionais a ele destinados"; por fim, o Artigo 25 determina que "o poder público apoiará a criação de universidade aberta para as pessoas idosas e incentivará a publicação de livros e periódicos, de conteúdo e padrão editorial adequados ao idoso, que facilitem a leitura, considerada a natural redução da capacidade visual". Embora estejam previstas oportunidades educacionais informais que consideram as necessidades e peculiaridades dessa fase da vida, são poucas as propostas educacionais oferecidas no Brasil que vão além da educação de jovens e adultos e/ou das universidades abertas para a terceira idade. 


\section{Considerações Finais}

A década de 1970 foi marcada tanto por um crescente número de estudos sobre a velhice, fase que começava a despertar interesse nas sociedades, bem como pelo aumento do número de trabalhos na área de Psicologia do Desenvolvimento cujo enfoque era o curso da vida. Nesse contexto, a perspectiva Life-Span contribuiu para a desmistificação do idoso como um ser doente e passivo, rompendo com a visão antagônica entre desenvolvimento e envelhecimento que vigorou até meados do século passado. Ela também chamou a atenção dos pesquisadores para a possibilidade de um envelhecimento saudável e funcional, enfatizando o caráter heterogêneo do mesmo.

Aliada ao crescente aumento mundial dessa população, a perspectiva Life-Span tem despertado a atenção de pesquisadores para os aspectos relacionados ao funcionamento físico, social e intelectual saudável na velhice. A educação, devido às irrefutáveis contribuições em todos esses âmbitos, tem-se destacado dentre as estratégias de promoção do desenvolvimento nessafase.

Não obstante, a educação de idosos pode ser considerada um tema recente entre os pesquisadores, educadores, legisladores e outros profissionais, especialmente no Brasil. A variedade de termos encontrados na literatura para designar o processo educacional ao longo do curso da vida corrobora a afirmação anterior. É evidente a incipiência da educação nessa fase, principalmente caso se considere o fato de que o estudo do envelhecimento começou a se desenvolver de forma mais sistematizada na década de 1970. O estado atual da área torna fundamental que se produza conhecimento científico que sirva como base para o desenvolvimento, a implantação e a avaliação de programas educacionais para idosos.

Conhecer os interesses da população que envelhece, assegurar seus direitos e viabilizar programas que sejam acessíveis à maioria é um imperativo social. Ressalta-se, por fim, que o envelhecimento populacional, a crescente demanda por atividades educacionais e o acúmulo de evidências de que é possível educar para um envelhecimento saudável constituem justificativas peremptórias para que sejam promovidas, com urgência, iniciativas efetivas de educação na

654 velhice.

\section{Referências}

Aramaki, F. O., \& Yassuda, M. S. (2011). Cognitive training based on metamemory and mental images: follow-up evaluation and booster training effects. Dementia \& Neuropsychologia, 5 (1), 48-53.

Baltes, P. B. (1987). Theoretical propositions of life-span developmental psychology: on the dynamics between growth and decline. Developmental Psychology, 32 (5), 611-626.

Baltes, P. B., \& Baltes, M. M. (1990). Psychological perspectives on successful aging: the model of selective optimization with compensation. In P. B. Baltes \& M. M. Baltes (Eds.), Successful aging: perspectives from behavioral sciences (pp.1-34). Cambridge: Cambridge University Press.

Baltes, P. B., Reese, H. W., \& Lipsitt, L. P. (1980). Life-span developmental psychology. Annual Review of Psychology, 31, 65-110.

Batistoni, S. S. T., Ordonez, T. O., Silva, T. B. L., Nascimento, P. P. P., Kissaki, P. T., \& Cachioni, M. (2011). Depressive symptoms in elderly participants of an open university for elderly. Dementia \& Neuropsychologia, 5 (2), 85-92.

Boulton-Lewis, G. M., Buys L., Lovie-Kitchin J., Barnett K., \& David N. (2007). Ageing, learning, and computer technology in Australia. Educational Gerontology, 33 (3), 253-270.

Brasil. (2003, 1 de outubro). Lei n. 10.741, de 1 de outubro de 2003. Dispõe sobre o estatuto do idoso. Brasília: Senado Federal.

Brasil. (1996, 23 de dezembro). Lei n. 9.394, de 20 de dezembro de 1996. Estabelece as diretrizes e bases da educação nacional. Recuperado em janeiro 20, 2008, disponível em <http://www.portal.mec.gov.br/arquivos/ $\mathrm{pdf} / \mathrm{ldb} . \mathrm{pdf}>$

Brasil. Ministério da Educação. (2000). Educação de jovens e adultos. Recuperado em janeiro 20, 2008, disponível em <http://portal.mec.gov.br/secad/>.

Brum, P. S., Forlenza, O. V., \& Yassuda, M. S. (2009). Cognitive training in older adults with mild cognitive impairment: impact on cognitive and functional performance. Dementia \& Neuropsychologia, 3 (2), 124-131.

Cachioni, M. (1998). Envelhecimento bem-sucedido e participação numa Universidade para a Terceira Idade: a experiência dos alunos da Universidade São Francisco. Dissertação de mestrado não-publicada, Faculdade de Educação, Universidade Estadual de Campinas.

Cachioni, M. (2005). Gerontologia educacional educação gerontológica. In A. L. Neri. Palavras-chave em gerontologia (pp.93-95). Campinas: Alínea.

Cachioni, M. (2007). Programas de educação permanente e reintegração social: a experiência das Universidades da Terceira Idade. In O. V. Forlenza. Psiquiatria geriátrica: do diagnóstico precoce à reabilitação (pp.391-398). São Paulo: Atheneu.

Cachioni, M. (2008). Universidade da Terceira Idade. In A. L. Neri (Org.), Palavras-chave em gerontologia (pp.207-210). Campinas: Alínea. 
Di Pierro, M. C. (2010). Notas sobre a redefinição da identidade e das políticas públicas de educação de jovens e adultos no Brasil. Educação \& Sociedade, 26 (92), 1115-1139.

Duay, D. L., \& Bryan, V. C. (2006). Senior adult's perceptions of successful aging. Educational Gerontology, 32 (6), 423-445.

Duay, D. L., \& Bryan, V. C. (2008). Learning in later life: what seniors want in a learning experience. Educational Gerontology, 34 (12), 1070-1086.

Fillit, H. M., Butler, R. N., O'Connell, A. W., Albert, M. S., Birren, J. E., Cotman, C. W., et al. (2002). Achieving and maintaining cognitive vitality with aging. Mayo Clinic Proceedings, 77 (7) 681-696.

Glendenning, F. (1989). Educational gerontology in Britain as an emerging field of study and practice. Educational Gerontology, 15 (2), 121-131.

Instituto Brasileiro de Geografia e Estatística. (2008). Síntese dos indicadores sociais de 2008. Recuperado em outubro 25, 2010, disponível em <http://www.ibge.gov.br/>.

Irigaray, T. Q., \& Schneider, R. H. (2008). Participação de idosas em uma universidade da terceira idade: motivos e mudanças ocorridas. Psicologia: Teoria e Pesquisa, 24 (2), 211-216.

Lemieux, A., \& Martinez, M. S. (2000). Gerontagogy beyond words: a reality. Educational Gerontology, 26 (5), 475-498.

Lima, M. P. (2000). Gerontologia educacional: uma pedagogia específica para o idoso, uma nova concepção develhice. São Paulo: LTr.

Lima-Silva, T. B., Ordonez, T. N., Santos, G. D. Fabrício, A. T., Aramaki, F. O., Almeida, E. B., et al. (2010). Effects of cognitive training based on metamemory and mental images. Dementia \& Neuropsychologia, 4 (2),114-119.

Marques, D. T., \& Pachane, G. G. (2010). Formação de educadores: uma perspectiva de educação de idosos em programas de EJA. Educação e Pesquisa, 36 (2), 475-490.

Minayo, M. C., Souza E. R., \& Paula D. R. (2010). Revisão sistemática da produção acadêmica brasileira sobre causas externas e violências contra a pessoa idosa. Ciência e Saúde Coletiva, 15 (6), 2719-2728.

Neri, A. L. (1995). Psicologia do envelhecimento: uma área emergente. In A. L. Neri (Org.), Psicologia do envelhecimento (pp.13-40). Campinas: Papirus.

Neri, A. L. (2001). Paradigmas contemporâneos sobre o desenvolvimento humano em psicologia e sociologia. In A. L. Neri (Org.), Desenvolvimento e envelhecimento: perspectivas biológicas, psicológicas e sociológicas (pp.11-37). Campinas: Papirus.

Neri, A. L. (2006). O legado de Paul B. Baltes à psicologia do desenvolvimento e do envelhecimento. Temas em Psicologia, $14(1), 17-34$.

Neri, A. L., \& Cachioni, M. (1999). Velhice bem-sucedida e educação. In A. L. Neri \& G. G. Debert (Orgs.), Velhice e sociedade (pp.113-140). Campinas: Papirus.

Ordonez, T. N., Yassuda, M. S., \& Cachioni, M. (2011). Elderly online: effects of a digital inclusion program in cognitive performance. Archives of Gerontology and Geriatrics, 53 (2), 216-219.

Palma, L. A., \& Cachioni, M. (2002). Educação permanente: perspectiva para o trabalho educacional com o adulto maduro e com o idoso. In E. Freitas, L. Py, A. L. Neri, F. A. X. Cançado, M. L. Gorzoni \& S. M. Rocha. Tratado de geriatria e gerontologia (pp.1101-1109). Rio de Janeiro: Guanabara Koogan.

Papaléo Netto, M. (2002). Estudo da velhice no século XX: histórico, definição do campo e termos básicos. In E. Freitas, L. Py, A. L. Neri, F. A. X. Cançado, M. L. Gorzoni \& S. M. Rocha. Tratado de geriatria e gerontologia (pp.2-12). Rio de Janeiro: Guanabara Koogan.

Portero, C. F., \& Oliva, A. (2007). Social support, psychological well-being, and health among the elderly. Educational Gerontology, 33 (12), 1053-1068.

Posada, F. V. (2004). Educación y personas mayores: algunas claves para la definición de uma psicologia de la educación em la vejez. Revista Brasileira de Ciências do Envelhecimento Humano, 1 (2), 61-76.

Ravelli, A. P. X., Fernandes, G. C. M., Barbosa, S. F. F., Simão, E., Santos, S. M. A., \& Meirelles, B. H. S. (2009). A produção do conhecimento em enfermagem e envelhecimento: estudo bibliométrico. Texto \& Contexto Enfermagem, 18 (3), 506-512

Russell, H. (2008). Later life: a time to learn. Educational Gerontology, 34 (3), 206-224.

Schaie, K. W. (1979). Cognitive development in aging. Retrieved on January 20, 2008, from <http://www.geron.psu.edu/>.

Staudinger, U. S., Marsiske, M., \& Baltes, P. B. (1993). Resilience and levels of reserve capacity in later adulthood: perspectives from life-spam theory. Development and Psychology, 5 (4), 541-566.

Swindell, R., \& Thompson, J. (1995). An international perspective on the University of the Third Age. Educational Gerontology, 21 (5), 429-447.

Todaro, M. A. (2005). Educação continuada: educação permanente. In A. L. Neri. Palavras-chave em gerontologia (pp.63-67). Campinas: Alínea.

Veras, R. (2009). Envelhecimento populacional contemporâneo: demandas, desafios e inovações. Revista de Saúde Pública, 43 (3), 548-554.

Webber, F., \& Celich, K. L. S. (2007). As contribuições da universidade aberta para a terceira idade no envelhecimento saudável. Estudos Interdisciplinares sobre Envelhecimento, 12, 127-142.

Yassuda, M. S., \&Silva, H. S. (2010). Participação em programas para a terceira idade: impacto sobre a cognição, humor e satisfação com a vida. Estudos de Psicologia (Campinas), 27 (2), 207-214. doi: 10.1590/S0103-166X201000020008.

Recebido em: 3/1/11

Versão final em: 23/3/2012

Aprovado em: 14/4/2012 
